\title{
Tremor control after pallidotomy in patients with Parkinson's disease: correlation with microrecording findings
}

Jamal M. Taha, M.D., Jacques Favre, M.D., Thomas K. Baumann, Ph.D., and Kim J. Burchiel, M.D.

Division of Neurosurgery and Department of Physiology and Pharmacology, Oregon Health Sciences University, Portland, Oregon

The goals of this study were to analyze the effect of pallidotomy on parkinsonian tremor and to ascertain whether an association exists between microrecording findings and tremor outcome.

Forty-four patients with Parkinson's disease (PD) who had drug-induced dyskinesia, bradykinesia, rigidity, and tremor underwent posteroventral pallidotomy. Using a 1- $\mu$-tip tungsten electrode, microrecordings were obtained through one to three tracts, starting $10 \mathrm{~mm}$ above the pallidal base. Tremor severity was measured on a patient-rated, 100-mm Visual Analog Scale (VAS), both preoperatively and 3 to 9 months (mean 6 months) postoperatively.

Preoperatively, tremor was rated as $50 \mathrm{~mm}$ or greater in 24 patients $(55 \%)$ and as less than $25 \mathrm{~mm}$ in 13 patients (30\%). Postoperatively, tremor was rated as $50 \mathrm{~mm}$ or greater in five patients $(11 \%)$ and less than $25 \mathrm{~mm}$ in 29 patients $(66 \%)$. The difference was significant $(\mathrm{p}=0.0001)$. Four patients $(9 \%)$ had no postoperative tremor. Tremor improved by at least $50 \%$ in eight $(80 \%)$ of 10 patients in whom tremor-synchronous cells were recorded (Group A) and in 12 (35\%) of 34 patients in whom tremor-synchronous cells were not recorded (Group B). This difference was significant $(\mathrm{p}=0.03)$. Tremor improved by at least $50 \mathrm{~mm}$ in all $(100 \%)$ of the seven Group A patients with severe $(>=50 \mathrm{~mm})$ preoperative tremor and in nine (53\%) of 17 Group B patients with severe preoperative tremor. This difference was also significant $(\mathrm{p}=0.05)$.

The authors proffer two conclusions: 1) after pallidotomy, tremor improves by at least $50 \%$ in two-thirds of patients with PD who have severe ( $>=50 \mathrm{~mm}$ on the VAS) preoperative tremor; and 2) better tremor control is obtained when tremor-synchronous cells are included in the lesion.

Key Words * microrecording * pallidotomy * Parkinson's disease * tremor

Thalamotomy has been the preferred surgical treatment for parkinsonian tremor for the past 30 years. $[3,8,10]$ Tremor is abolished in 86 to $95 \%$ of patients with Parkinson's disease (PD) after this procedure; $[3,8,12,16]$ however, the effects of thalamotomy on other symptoms of PD (that is, bradykinesia, rigidity, dystonia, drug-induced dyskinesia, and on-off fluctuations) have been inconsistent.[3,8] For amelioration of the latter symptoms, posteroventral pallidotomy has proved to be a more effective surgery.[2,5,9,11,14] 
Currently, pallidotomy is the preferred treatment for most symptoms of PD.[2,5,9,11,14] Authors of several series have reported that, in addition to improvement of drug-induced dyskinesia, bradykinesia, and rigidity, pallidotomy also improves tremor in more than 80 to $85 \%$ of patients with PD.[9,11] However, the authors of those series did not fully analyze the degree of tremor improvement after pallidotomy. This information is important to define more clearly the surgical indications for pallidotomy versus thalamotomy in patients with PD who suffer from severe tremor. In addition, none of these authors analyzed factors associated with tremor improvement after pallidotomy. Specifically, there has been no detailed analysis of the association between microrecording findings and postoperative tremor control, similar to the analysis that demonstrated such an association for thalamotomy.[12,16]

In this study, we analyzed tremor control after pallidotomy in 44 patients with PD and investigated whether an association exists between intraoperative microrecording findings and postoperative tremor control.

\section{CLINICAL MATERIAL AND METHODS}

\section{Patient Population}

In 1995, 44 patients suffering from PD underwent pallidotomy using microrecording techniques at the Oregon Health Sciences University in Portland, Oregon. Informed consent was obtained from all patients. There were 26 men and 18 women, aged 42 to 78 years. Twenty-five patients underwent unilateral pallidotomy and 19 patients had simultaneous bilateral pallidotomy. All patients exhibited poor control of PD symptoms with dopaminergic medications either because of decreased efficacy or severe "on-off" fluctuations. Selection criteria for surgery included initial response to levodopa and uncontrolled dyskinesia, dystonia, rigidity, and bradykinesia. Although resting tremor was present in all patients, it was not the sole indication for surgery in any patient. None of the patients had "Parkinson's plus" syndromes or suffered from severe dementia.

\section{Surgical Technique}

Pallidotomy was performed using a Leksell stereotactic system (Elekta Instruments, Atlanta, GA) guided by magnetic resonance (MR) imaging. All surgical procedures were performed after the patient had received a local anesthetic, without intraoperative sedation and with the patient in the "off" cycle. The stereotactic frame was affixed to the patient's head, guided by the ear bars and known external landmarks,[18] which positioned the frame parallel to the intercommissural plane. Magnetic resonance imaging was performed using a 1.5-tesla imaging system (Signa; General Electric Medical Systems, Milwaukee, WI). Sagittal, coronal, and axial images parallel to the intercomissural plane were obtained. Proper frame placement and imaging planes (that is, orthogonal to the intercommissural plane) were verified. The pallidal target was measured $2 \mathrm{~mm}$ anterior to the midcommissure, 3 to $6 \mathrm{~mm}$ below the intercommissural line, and 19 to $22 \mathrm{~mm}$ lateral to the midline, just lateral to the optic tract. The stereotactic coordinates of the pallidal target were calculated using built-in MR software and verified using the functional digitizer (Elekta Instruments).

After assembling the stereotactic arc system onto the stereotactic frame, a 3-mm twist-drill hole was created approximately $2 \mathrm{~cm}$ lateral to the midline, just anterior to the coronal suture. The trajectory to the pallidal target in the sagittal plane was $63 \pm 14$ š to the intercommissural line; the trajectory in the coronal plane was $90 \pm 3$ š to the midsagittal plane. 


\section{Microrecording Technique and Signal Analysis}

Extracellular single unit discharges were recorded starting $10 \mathrm{~mm}$ above the MR-calculated pallidal target and through the pallidal base. Microrecordings were obtained through one to three trajectories, 2 $\mathrm{mm}$ apart, for each patient. Each trajectory was created using a separate twist-drill hole. Single-unit discharges were recorded extracellularly using specially designed, monopolar tungsten microelectrodes that had diameter tips of 1 to $2 \mu \mathrm{m}$ and impededances of 1.1 to $1.4 \mathrm{Mohm}$ at $1000 \mathrm{~Hz}$.[13] The microelectrode sliding cannula was used as the indifferent electrode. The microelectrode was advanced in micrometer increments using a manual hydraulic microdrive (model 51421; Stoelting, Wood Dale, IL). A unity-gain preamplifier (High Impedance Probe; Grass-Astromed, Warwick, RI), which was affixed to the stereotactic arc, transmitted the signal potentials from the microelectrode to an alternating current-coupled differential amplifier (P511; Grass-Astromed). The action potential signals were filtered using a bandwidth of 300 to $10,000 \mathrm{~Hz}$ and amplified 10,000 times. The signal was displayed on an oscilloscope with Schmidt-trigger capabilities (Tektronix, Beaverton, OR) and stored on magnetic tape. The signal was also connected to an audio monitor. The patient's movements during microrecording were continuously monitored using a video camera. Tremor activity was recorded using an accelerometer.

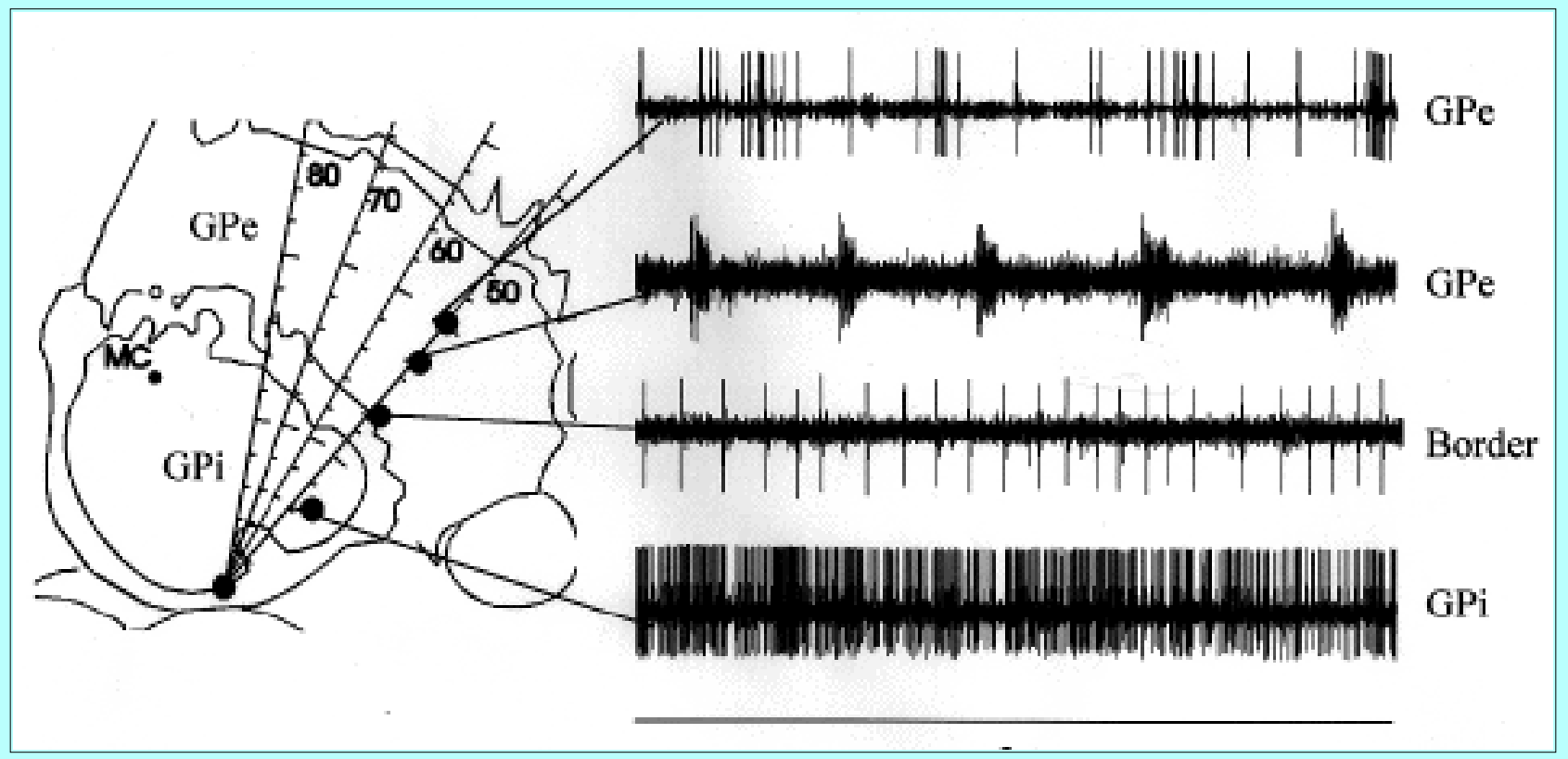

Fig. 1. Digitized display of microrecordings (right) of typical external pallidal (GPe), internal pallidal (GPi), and border cells and their corresponding locations (left) on a sagittal section of the globus pallidus, $21.5 \mathrm{~mm}$ lateral to the midline. $\mathrm{MC}=$ midcommissure. $\mathrm{Bar}=$ 2 seconds.

Single unit discharges were identified intraoperatively by their typical firing frequencies and amplitudes and verified using Schmidt-triggering techniques (Fig. 1). The characteristics of the recorded cells were analyzed using a personal computer software package that has spike amplitude discriminating capabilities. The software allowed the construction of interspike interval histograms and spike count-per-unit time histograms. Neurons of the external pallidal segment (GPe) were identified by their typical irregular and/or short-burst activity, whereas neurons of the internal pallidal segment (GPi) were identified by their typical sustained, high-frequency discharges.[4-7] Border cells were identified by their typical low-frequency, relatively tonic discharges.[4-7] Kinesthetic cells were identified by the change in their firing rate or pattern in response to passive movements of the contralateral and ipsilateral limbs. The pallidal base was identified by the absence of GPi neuronal action potentials and by the change in 
background neuronal noise. Optical tract recordings were performed in the last 16 patients using the technique described by Lozano, et al.[13]

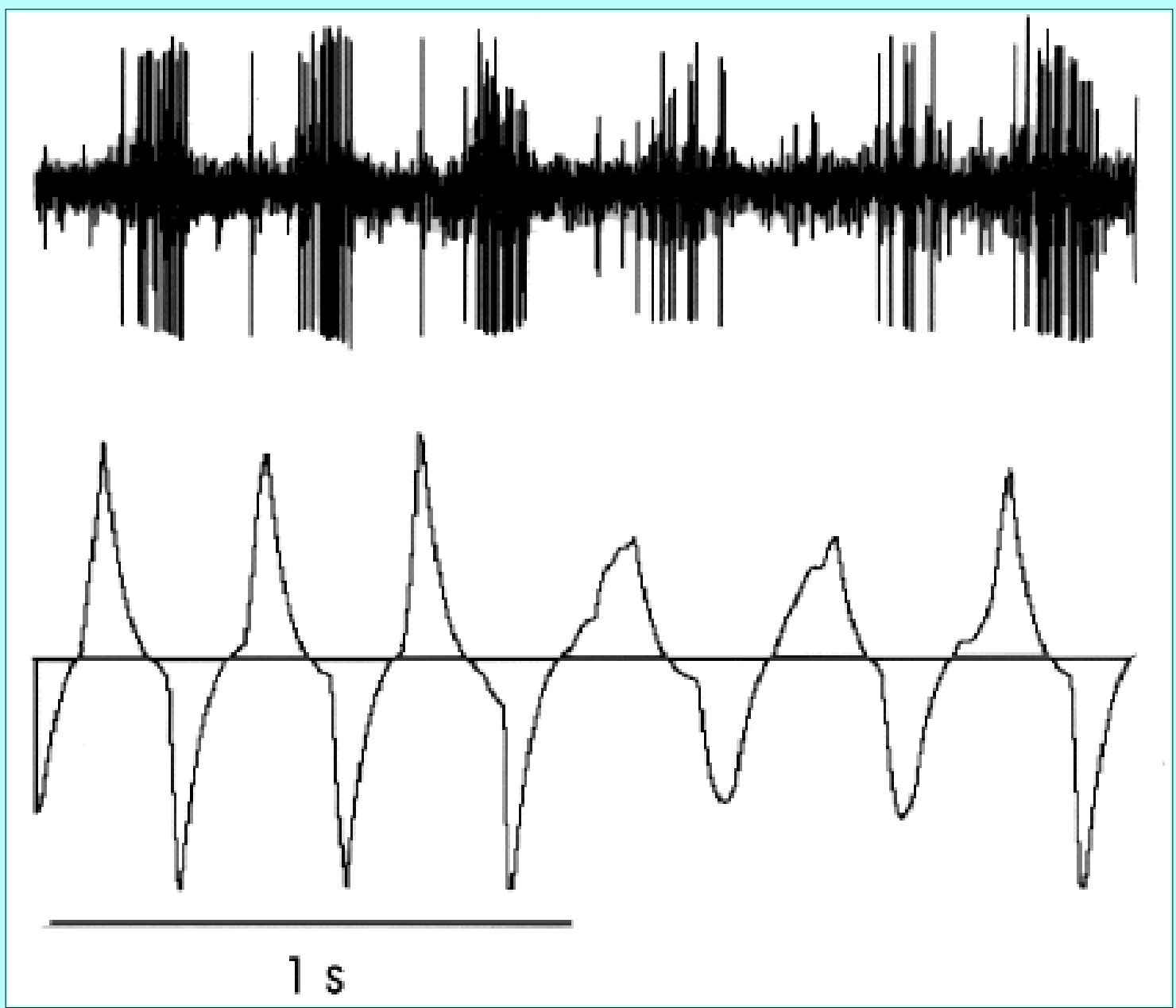

Fig. 2. Digitized display of a tremor-synchronous cell. Upper trace represents signal action potential; lower trace depicts the corresponding tremor activity recorded by an accelerometer. Bar $=1$ second.

Neurons that discharged at a tremor-synchronous frequency $(3-7 \mathrm{~Hz})$ were tested for responses to joint movements. Tremor-synchronous cells were defined as cells that fired in synchrony with the tremor, including cells that responded to contralateral limb movement and cells in which no kinesthetic receptive field could be identified (Fig. 2). All cells, including tremor-synchronous cells, were plotted on the corresponding sagittal sections of the globus pallidus obtained from the Schaltenbrand and Bailey stereotactic atlas[17] to determine their spatial localization.

\section{Lesion Production}

Using a 1.3 X 2-mm electrode (Radionics, Inc., Burlington, MA), macrostimulation (1-msec square wave) was performed at $2 \mathrm{~Hz}$ and $50 \mathrm{~Hz}$ to confirm the appropriate location of the target. A preliminary lesion was created at $84 \check{\mathrm{s}} \mathrm{C}$ for 10 seconds to confirm the absence of side effects (weakness, visual field defect, or speech difficulty). The final pallidotomy lesion consisted of three thermal lesions, linearly spaced $2 \mathrm{~mm}$ apart along the same trajectory. The final lesion closely resembled the shape of a cylinder that encompassed GPi and possibly the inferomedial portion of GPe. Each thermal lesion was created by emitting a temperature of $84 \check{s} \mathrm{C}$ for 60 seconds, starting at the pallidal base. If kinesthetic and/or tremor-synchronous cells were recorded above $6.5 \mathrm{~mm}$ from the base of the pallidum, a fourth thermal 
lesion was added.

\section{Postoperative Follow-Up Evaluation and Data Analysis}

Patients were kept on the same antiparkinsonian medications for 1 month postoperatively, after which dosages were adjusted based on the patient's signs and symptoms. All patients were asked preoperatively to rate the severity of their tremor on a Visual Analog Scale (VAS) that ranged from 0 to $100 \mathrm{~mm}(0 \mathrm{~mm}$ $=$ no tremor, $100 \mathrm{~mm}=$ worst possible tremor), similar to that used for pain assessment.[15] Patients also rated their tremor 3 to 9 months (mean 6 months) postoperatively. There were two reasons why we chose to use a subjective VAS score for tremor assessment. First, many patients lived far away and could not return for formal testing. Second, many of our patients did not have a tremor that persisted at the same severity throughout the day, depending on their intake of medication and general activity. This made one-time testing in the office an inaccurate assessment of the severity of the tremor. To reduce a placebo effect, we resorted to three procedures: first, patients assessed their postoperative tremor at least 3 months after the procedure. Second, patients assessed their postoperative tremor without reviewing their preoperative assessment. Third, families were requested to verify preoperative and postoperative tremor assessment. We have been routinely using VAS scores in addition to formal neurological testing to assess the results of pallidotomy on all parkinsonian symptoms and have found a close correlation of approximately $90 \%$. However, this is the subject of another communication. Microrecording data (videotape, data sheets, and neuronal maps) were reviewed for each patient for the presence and localization of tremor-synchronous cells.

Tremor was considered severe if it measured at least $50 \mathrm{~mm}$ on the VAS, moderate if it measured between 25 and $50 \mathrm{~mm}$, and mild if it measured less than $25 \mathrm{~mm}$. Postoperative improvement was considered good if the tremor improved by more than 50\%, fair if it improved by 25 to $50 \%$, and poor if it improved by less than $25 \%$. All results were statistically analyzed using chi-square analysis with Yates' correction for data values greater than 5 and Fisher's exact test for data values less than 5.[1] Significance was accepted for probability values of less than 0.05 .

\section{RESULTS}

Analysis was performed for two main parameters: postoperative tremor outcome and the association between tremor-synchronous cells and postoperative tremor relief.

\section{Postoperative Tremor Outcome}

Of the 44 patients with PD, preoperatively $24(55 \%)$ had severe tremor, seven (16\%) had moderate tremor, and $13(30 \%)$ had mild tremor. Postoperatively, five patients $(11 \%)$ had severe tremor, $10(23 \%)$ had moderate tremor, and $29(66 \%)$ had mild tremor (Table 1), a difference that is statistically significant. Four patients $(9 \%)$ had complete abolition of tremor after pallidotomy. 


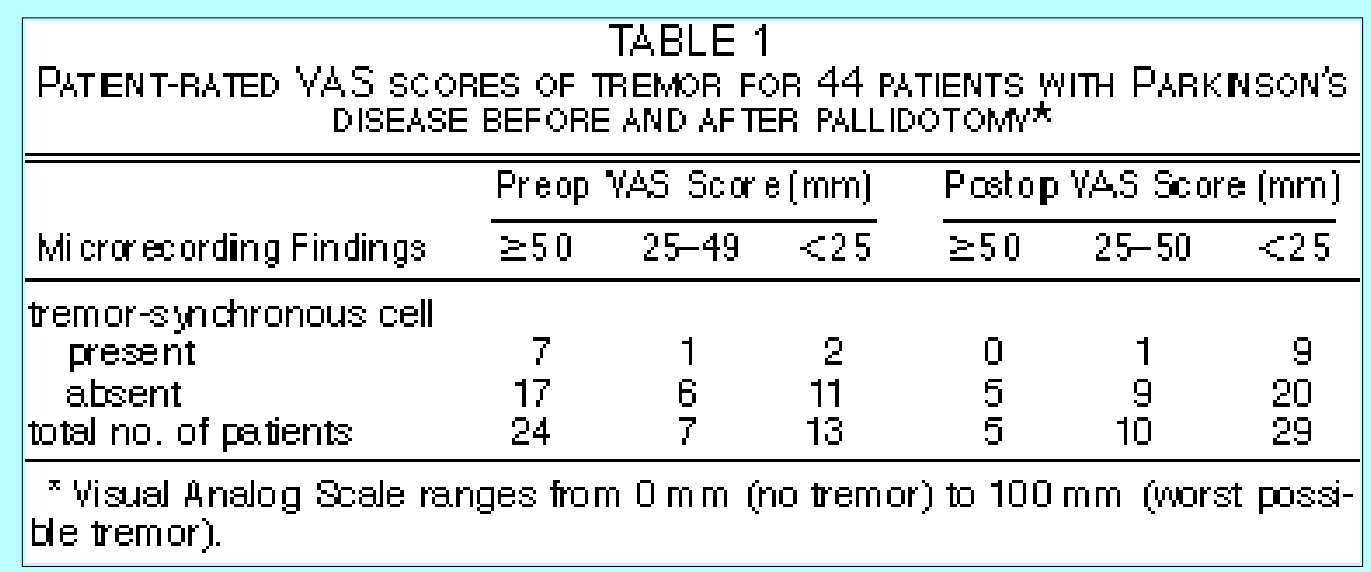

The degree of postoperative tremor improvement was calculated for all patients (Table 2). Of 44 patients, $20(45 \%)$ identified good improvement, $10(23 \%)$ had fair improvement, and $14(32 \%)$ had poor or no improvement. Of 24 patients with severe ( $>=50 \mathrm{~mm}$ VAS score) preoperative tremor, 16 patients $(67 \%)$ had their tremor improve by a VAS score of at least $50 \mathrm{~mm}$, five patients $(21 \%)$ had tremor improvement of $25 \mathrm{~mm}$ to $50 \mathrm{~mm}$, and three patients $(12 \%)$ had changes in tremor that measured less than $25 \mathrm{~mm}$ or no improvement.

\begin{tabular}{|c|c|c|c|c|c|c|}
\hline \multirow[b]{3}{*}{ Mi crơ es ording Findings } & \multicolumn{3}{|c|}{$\begin{array}{l}\text { TABLE } 2 \\
\text { T IN TREM }\end{array}$} & $\mathrm{DBV}$ & $\mathrm{Ssc}$ & \\
\hline & \multicolumn{3}{|c|}{ Percentage Im proven ent } & \multicolumn{3}{|c|}{$\begin{array}{c}\text { A.tsolute } \\
\text { Im provenent ( } \mathrm{mm})\end{array}$} \\
\hline & $\geqq 50$ & $25-49$ & $<25$ & $\geqq 50$ & $25-49$ & $<25$ \\
\hline \multicolumn{7}{|l|}{ tremor-synchronous cell } \\
\hline present & 8 & 0 & 2 & 7 & 1 & 2 \\
\hline absent & 12 & 10 & 12 & 9 & 7 & 18 \\
\hline total no. of patients & 20 & 10 & 14 & 16 & 8 & 20 \\
\hline
\end{tabular}

\section{Association Between Tremor-Synchronous Cells and Postoperative Tremor Relief}

For the purpose of comparison, patients in whom tremor-synchronous cells were recorded were identified as Group A, whereas patients in whom tremor-synchronous cells were not recorded were identified as Group B. Of the 44 patients with PD, 10 (23\%) had recordings of tremor-synchronous cells (Group A) and $34(77 \%)$ did not (Group B). Of the 10 patients in Group A, preoperatively seven (70\%) had severe tremor, one $(10 \%)$ had moderate tremor, and two (20\%) had mild tremor. Postoperatively, none (0\%) of the patients in Group A described severe tremor, one (10\%) had moderate tremor, and nine (90\%) had mild tremor (Table 1). This difference is statistically significant. Of the 34 patients in Group B, preoperatively $17(50 \%)$ had severe tremor, six (18\%) had moderate tremor, and $11(32 \%)$ had mild tremor (Table 1). Postoperatively, five (15\%) of the Group B patients had severe tremor, nine (26\%) had moderate tremor, and $20(59 \%)$ had mild or no tremor (Table 1). Again, this difference is statistically significant.

The degree of postoperative tremor improvement was calculated for both groups (Table 2). Of the 10 patients in Group A, eight (80\%) had good improvement, none $(0 \%)$ had fair improvement, and two (20\%) had poor or no improvement. Of the 34 patients in Group B, 12 (35\%) had good improvement, 10 
(30\%) had fair improvement, and $12(35 \%)$ had poor or no improvement. The difference is statistically significant. Of the seven Group A patients who had severe preoperative tremor, all (100\%) had postoperative tremor improvement that measured at least $50 \mathrm{~mm}$ on the VAS. Of the 17 Group B patients who had severe preoperative tremor, nine (53\%) had postoperative tremor improvement that measured at least $50 \mathrm{~mm}$ on the VAS. The difference is statistically significant.

\section{DISCUSSION}

This study documents that pallidotomy improves parkinsonian tremor in most patients, but rarely (9\% in our series) abolishes it. Our findings also suggest that inclusion of cells that fire in synchrony with tremor is associated with better postoperative tremor control.

\section{Postoperative Tremor Outcome}

Several clinical series have documented improvement in parkinsonian tremor after pallidotomy. Laitinen, et al.,[11] reported excellent or good tremor relief in $81 \%$ of 32 patients. Although these researchers concluded that tremor could be effectively abolished by pallidotomy, they gave no analysis of the severity of the residual tremor. At least nine (28\%) patients had persistent tremor activity, which was severe in six (19\%). Iacono, et al.,[9] reported improvement in tremor in 85\% of 126 patients with PD after pallidotomy. Tremor improved by $65 \%$ from mean scores (on the Unified Parkinson's Disease Rating Scale) of 2.2 preoperatively to 0.73 postoperatively. However, it was not clear how many patients had abolition of tremor after surgery or how many patients had persistent significant tremor that interfered with daily activities and/or required further treatment.

In our series of 44 patients, moderate or severe tremor was found in 31 patients (70\%) preoperatively compared to 15 patients (34\%) postoperatively (Table 1). This difference is statistically significant. Tremor decreased by more than half in $20(45 \%)$ of 44 patients. Of the 24 patients with severe preoperative tremor, $16(67 \%)$ cited postoperative tremor improvement of at least $50 \%$ and at least 50 $\mathrm{mm}$ on the VAS; only five (21\%) of the 24 patients suffered from persistent severe tremor postoperatively. Of the 44 patients, four $(9 \%)$ had tremor abolition after surgery. These results are somewhat similar to those reported in other series.[2,5,9,11,14] Our analysis of data provided in the literature $[2,5,9,11,14]$ and the results of this series yield one conclusion: tremor improves to various degrees in most patients after pallidotomy, but it is infrequently abolished. We do not believe that limitations of this study, such as short-term follow up and subjective tremor scale assessment, alter this conclusion.

\section{Association Between Tremor-Synchronous Cells and Postoperative Tremor Relief}

After thalamotomy, better tremor control has been reported when cells that fire in synchrony with tremor are identified during surgery and included in the thermal lesion.[12,16] Our findings suggest that such a correlation may also exist for pallidotomy. In our series, tremor decreased by more than half in eight $(80 \%)$ of 10 patients in whom tremor-synchronous cells were isolated, compared to $12(35 \%)$ of 34 patients in whom tremor-synchronous cells were not isolated. Tremor improved by at least $50 \mathrm{~mm}$ in the VAS in all (100\%) of the seven patients who had severe preoperative tremor and in whom tremor-synchronous cells were recorded, compared to nine (53\%) of the 17 patients who had preoperative severe tremor and in whom tremor-synchronous cells were not recorded. The differences are statistically significant. These results indicate that better postoperative tremor control is achieved if tremor-synchronous cells are included in the lesion; however, we consider these results to be preliminary 
because of the small sample size and the limited follow-up period. It is important to note that based on our results we cannot conclude that use of microrecroding techniques improves tremor control after pallidotomy. Our results merely suggest that better tremor control is achieved when tremor-synchronous cells are present and when they are included in the lesion.

It is important to mention that it may not be easy to differentiate true "tremor cells" from kinesthetic cells that discharge in bursts in response to oscillatory limb movement. Such a differentiation is sometimes possible by pretrigger spike averaging techniques. In our series, we did not attempt such a differentiation and defined all cells with burst discharges at 3 to $8 \mathrm{~Hz}$ in synchrony with tremor as tremor-synchronous cells, regardless of the presence of kinesthetic receptive fields. This means that the tremor-synchronous cells we defined could represent cells that drive tremor, cells that respond to tremor, or a combination of both cells. In the GPi of African green monkeys, cells that discharge in bursts at 4 to $8 \mathrm{~Hz}$ increased from $0.6 \%$ in normal animals to $25 \%$ in animals treated by the neurotoxin

1-methyl-4-phenyl-1,2,3,6-tetrahydropyridin.[6] The latter finding implies that such discharge patterns are pathological in PD and one may speculate that by including these cells in the thermal lesion, tremor would subside. Based on our results, we cannot prove that such a cause-and-effect relationship exists. Tremor improvement in patients in whom tremor-synchronous cells were identified could represent a mere association between a certain pathological state and outcome. In addition, it is not clear whether a paucity of tremor-synchronous cells represents a different pathological state, inaccurate target localization, or mere absence of such cells in a specific trajectory (most recordings were performed through one trajectory). Regardless of the true nature of the association, our results demonstrate that tremor was better improved after pallidotomy if tremor-synchronous cells were recorded during surgery and were included in the lesion than if they were not.

\section{Selection of Surgery for Parkinsonian Tremor}

The fact that tremor improved but did not, in most patients, subside after pallidotomy implies that the olivocerebellopontine system may play a larger role in parkinsonian tremor than the corticosubthalamic-GPi-thalamic circuit. Based on our results and a review of the literature, the results of pallidotomy in relieving parkinsonian tremor are inferior to those of thalamotomy. After pallidotomy, 68 to $85 \%$ of patients have tremor improvement but tremor abolition is infrequent.[2,5,9,11,14] After thalamotomy, 85 to $95 \%$ of patients have tremor abolition. $[3,8,12,16]$ Based on these results, we recommend thalamotomy for patients with PD who have tremor as the predominant symptom for which they seek treatment, and pallidotomy for patients who have tremor in addition to other symptoms of PD, such as drug-induced dyskinesia, bradykinesia, and rigidity. However, it is not clear whether in patients with PD who have tremor as the predominant symptom, the functional outcome is very different between patients who achieve tremor improvement after pallidotomy versus patients who achieve tremor abolition after thalamotomy. Of particular consideration are patients with PD who take levodopa primarily to control tremor but who develop drug-induced dyskinesia. In these patients, thalamotomy will most likely abolish tremor and eliminate the need for levodopa intake and the associated dyskinesia. However, before the physician recommends thalamotomy for these patients, prescribed levels of levodopa should be decreased or discontinued to assess the presence and/or severity of other associated symptoms of PD, which may have been masked by the intake of levodopa. In patients in whom the other symptoms of PD have been masked by levodopa, pallidotomy will most likely relieve drug-induced dyskinesia and will allow the continuation of levodopa intake for the relief of tremor (and the other associated symptoms) if required. 


\section{CONCLUSIONS}

Based on the results of our study, we conclude the following: 1) after pallidotomy, two-thirds of patients with PD who have severe $(>=50$ score on the VAS) preoperative tremor have at least a $50 \%$ tremor improvement; 2) better tremor control (at least by half in all patients with severe tremor) may be obtained when tremor-synchronous cells are isolated and included in the lesion.

\section{References}

1. Armitage P, Berry G: Statistical Methods in Medical Research, ed 2. Oxford: Blackwell, 1987, pp $125-132$

2. Baron MS, Vitek JL, Bakay RAE, et al: Treatment of advanced Parkinson's disease by posterior GPi pallidotomy: 1-year results of a pilot study. Ann Neurol 40:355-366, 1996

3. Burchiel KJ: Thalamotomy for movement disorders. Neurosurg Clin North Am 6:55-71, 1995

4. DeLong MR, Crutcher MD, Georgopoulos AP: Primate globus pallidus and subthalamic nucleus: functional organization. J Neurophysiol 53:530-543, 1985

5. Dogali M, Fazzini E, Kolodny E, et al: Stereotactic ventral pallidotomy for Parkinson's disease. Neurology 45:753-761, 1995

6. Filion M, Tremblay L: Abnormal spontaneous activity of globus pallidus neurons in monkeys with MPTP-induced parkinsonism. Brain Res 547:142-151, 1991

7. Filion M, Tremblay L, Bédard P: Abnormal influences of passive limb movement on the activity of the globus pallidus neurons in parkinsonian monkeys. Brain Res 444:165-176, 1988

8. Fox MW, Ahlskog JE, Kelly PJ: Stereotactic ventrolateralis thalamotomy for medically refractory tremor in post-levodopa era Parkinson's disease patients. J Neurosurg 75:723-730, 1991

9. Iacono RP, Shima F, Lonser RR, et al: The results, indications, and physiology of posteroventral pallidotomy for patients with Parkinson's disease. Neurosurgery 36:1118-1125, 1995

10. Laitinen LV: Brain targets in surgery for Parkinson's disease. Results of a survey of neurosurgeons. $\mathbf{J}$ Neurosurg 62:349-351, 1985

11. Laitinen LV, Bergenheim AT, Hariz MI: Leksell's posteroventral pallidotomy in the treatment of Parkinson's disease. J Neurosurg 76:53-61, 1992

12. Lenz FA, Tasker RR, Kwan HC, et al: Single unit analysis of the human ventral thalamic nuclear group: correlation of thalamic "tremor cells" with the 3-6 Hz component of parkinsonian tremor. J Neurosci 8:754-764, 1988

13. Lozano A, Hutchison W, Kiss Z, et al: Methods for microelectrode-guided posteroventral pallidotomy. J Neurosurg 84:194-202, 1996

14. Lozano AM, Lang AE, Galvez-Jimenez N, et al: Effect of GPi pallidotomy on motor function in Parkinson's disease. Lancet 346:1383-1387, 1995 
15. Price DD, Bush FM, Long S, et al: A comparison of pain measurement characteristics of mechanical visual analogue and simple numerical rating scales. Pain 56:217-226, 1994

16. Quinones-Molina R, Molina H, Ohye C, et al: CT-oriented microrecording guided selective thalamotomy. Stereotact Funct Neurosurg 62:200-203, 1994

17. Schaltenbrand G, Bailey P: Introduction to Stereotaxis with an Atlas of the Human Brain. Stuttgart: Thieme, 1959

18. Spiegelmann R, Friedman W: Rapid determination of thalamic CT-stereotactic coordinates: a method. Acta Neurochir 110:77-81, 1991

Manuscript received August 2, 1996.

Accepted in final form November 19, 1996.

Address reprint requests to: Jamal M. Taha, M.D., Department of Neurosurgery, University of Cincinnati College of Medicine, 231 Bethesda Avenue, Cincinnati, Ohio 45267-0515. 